\title{
The Prevalence and Genetic Characterization of Human Metapneumovirus in Bulgaria, 2016-2019
}

\author{
Neli S. Korsun ${ }^{a}$ Svetla G. Angelova ${ }^{a} \quad$ Ivelina T. Trifonova ${ }^{a}$ Silvia E. Voleva ${ }^{a}$ \\ Iliana G. Grigorova ${ }^{a}$ Iren S. Tzotcheva ${ }^{b}$ Sirma D. Mileva ${ }^{b}$ Penka I. Perenovska ${ }^{b}$ \\ aDepartment of Virology, National Center of Infectious and Parasitic Diseases, Sofia, Bulgaria; bediatric Clinic, \\ University Hospital Alexandrovska, Medical University, Sofia, Bulgaria
}

\author{
Keywords \\ Respiratory infection · Respiratory virus · Human \\ metapneumovirus · Genetic diversity · Genotype · Amino \\ acid substitution
}

\begin{abstract}
Introduction: We investigated the prevalence of human metapneumovirus (hMPV) among patients with acute respiratory infections in Bulgaria, and performed genetic characterization of the F gene of these strains. Methods: Nasopharyngeal swabs collected from patients of a range of ages were tested by using real-time PCR for 12 respiratory viruses. The $F$ gene was sequenced, and phylogenetic and amino acid analyses of the F gene/protein were performed. Results: A total of 1,842 patients were examined during a 3-year period; 1,229 patients (66.7\%) were positive for at least one respiratory virus. hMPV was identified in 83 (4.5\%) patient samples. Eleven (13\%) of hMPV-positive patients were coinfected with another respiratory virus. The hMPV incidence rate in the 2016/2017,2017/2018, and 2018/2019 winter seasons was $5.4,5.4$, and $3.1 \%$, respectively. hMPV was mainly detected in specimens collected between January and May (89.2\% of cases). The incidence of hMPV infection was highest (5.1\%) among the youngest age-group ( $0-4$ years), where hMPV was a causative agent in 8.1 and $4.8 \%$ of bronchiolitis and pneumonia cases, respectively. Among the patients aged $\geq 5$ years, hMPV was detected in 2.2 and $3.2 \%$ of cases of pneumonia and central nervous system infections, re-
\end{abstract}

karger@karger.com
www.karger.com/int

Karger $\stackrel{\text { ' }}{5}$
(C) 2021 The Author(s)

Published by S. Karger AG, Basel

This is an Open Access article licensed under the Creative Commons Attribution-NonCommercial-4.0 International License (CC BY-NC) (http://www.karger.com/Services/OpenAccessLicense), applicable to the online version of the article only. Usage and distribution for commercial purposes requires written permission. spectively. Phylogenetic analysis of the $\mathrm{F}$ gene showed that the sequenced hMPV strains belonged to the $A 2 b, B 1$, and B2 genotypes. Numerous amino acid substitutions were identified compared with the NL00/1 prototype strain. Conclusion: This study revealed the significant role of hMPV as a causative agent of serious respiratory illnesses in early childhood, and also demonstrated year-to-year changes in hMPV prevalence and genetic diversity in circulating strains.

(C) 2021 The Author(s)

Published by S. Karger AG, Basel

\section{Introduction}

Acute respiratory infections (ARIs) are associated with high global morbidity and mortality, and represent a substantial health-care burden. Over 200 types of viruses from different families cause ARIs, and among these the human metapneumovirus (hMPV) has been identified as a major causative agent of acute upper and lower respiratory infections (ALRIs), especially in young children, the elderly, and immunocompromised patients [1-3]. Globally, >14.2 million hMPV-associated ALRI cases, 643,000 hospital admissions, 7,700 inhospital deaths, and 16,100 overall (hospital and community) deaths are estimated to annually occur in children younger than 5 years [4]. Currently, there are no licensed vaccines or antiviral drugs for preventing or treating hMPV infections. hMPV is very closely related to respiratory syncytial virus (RSV), and both viruses share similar structural, clinical, and epidemiological character- 
istics. These viruses belong to the family Pneumoviridae but to different genera: Orthopneumovirus (RSV) and metapneumovirus (hMPV) [5]. hMPV virions consist of a nonsegmented, single-stranded, negative-sense RNA genome of approximately $13.3 \mathrm{~kb}$ that encodes 9 proteins: nucleoprotein $(\mathrm{N})$, phosphoprotein $(\mathrm{P})$, matrix protein $(\mathrm{M})$, fusion protein $(\mathrm{F})$, matrix-2 proteins (M2-1 and M22), small hydrophobic protein ( $\mathrm{SH}$ ), attachment glycoprotein $(\mathrm{G})$, and a large polymerase protein $(\mathrm{L})$. The surface glycoproteins $\mathrm{F}$ and $\mathrm{G}$ are the major antigens, that induce protective neutralizing antibodies. The $\mathrm{G}$ protein enables attachment of the virus to target cells via interaction with cellular glycosaminoglycans. The $\mathrm{F}$ protein mediates attachment of the virus to integrins as cellular receptors, thereby allowing entry of the viral ribonucleoprotein into the cell cytoplasm by fusing of the viral envelope and cell membrane. The F protein forms a trimer, and is expressed as the F0 precursor that is cleaved by a cellular protein into 2 subunits, F1 and F2, which contain several functional domains $[6,7]$. Under host immune pressure, amino acid substitutions accumulate in viral surface glycoproteins, especially in the $G$ protein, leading to escape from preexisting immunity. Based on nucleotide sequence analysis of the $F$ and $G$ genes, hMPV is classified into 2 major genetic groups (A and $\mathrm{B}$ ), and these are further divided into 4 major genotypes (A1, A2, B1, and B2) and 2 additional genotypes (A2a and A2b) [6]. Recently, 3 novel hMPV subtypes $\mathrm{A} 2 \mathrm{~b} 1, \mathrm{~A} 2 \mathrm{~b} 2$, and $\mathrm{A} 2 \mathrm{c}$ have been proposed $[8,9]$. In a particular season, viruses from these different genotypes can co-circulate within the same region as the predominant genotypes can vary annually.

Despite the important clinical significance of hMPV infection, information regarding the prevalence of hMPV in Bulgaria is currently limited. Previous studies examining circulation patterns of hMPV in Bulgaria have focused on the under-5 age-group, and data are absent for prevalence among patients over the entire age range and on the molecular epidemiology of this pathogen [10-13]. The aims of this study were to investigate the epidemiological and clinical characteristics of hMPV infections among patients from different age-groups over a 3-year period, and to perform a phylogenetic and amino acid sequence analysis of the F gene/protein of hMPV strains.

\section{Materials and Methods}

\section{Patients and Specimen Collection}

The study population comprised patients of a range of ages from different regions of Bulgaria who had been treated for ARI in primary care settings or hospitals. ARIs were defined according to the European Centre for Disease Prevention and Control (https:// ecdc.europa.eu/en/infectious-diseases-public-health/surveillance-and-disease-data/eu-case-definitions). Patients were diagnosed by attending physicians based on standard clinical criteria. Combined nasopharyngeal and oropharyngeal specimens were collected from all enrolled patients using commercial polyester swabs (Deltalab, Barcelona, Spain) either during a physician visit or within the first $24 \mathrm{~h}$ of admission. The specimens were taken 1-7 days after the onset of illness. After collection, swabs were stored at $2-8^{\circ} \mathrm{C}$ for up to $72 \mathrm{~h}$ and transported in ice packs to the National Laboratory "Influenza and ARD," which is recognized as a World Health Organization National Influenza Center. Specimens were processed immediately for virus detection or stored at $-80^{\circ} \mathrm{C}$ prior to analysis.

\section{Molecular Detection of Respiratory Viruses}

Total viral nucleic acids were extracted automatically from a $700 \mu \mathrm{L}$ specimen aliquot and eluted in $75 \mu \mathrm{L}$ using the ExiPrep Dx Viral DNA/RNA kit and ExiPrep16DX equipment (Bioneer, Daejeon, South Korea). Influenza viruses were tested via a real-time RT-PCR method using the SuperScript III Platinum One-Step qRT-PCR system (Invitrogen, Thermo Fisher Scientific, Waltham, MA, USA). Primers and probes were provided by the International Reagent Resource, USA. Amplification was performed using a CFX96 thermal cycler (Bio-Rad Laboratories, Inc., Hercules, CA, USA) according to the protocol recommended by Centers for Disease Control and Prevention, Atlanta, GA, USA (reverse transcription at $50^{\circ} \mathrm{C}$ for $30 \mathrm{~min}$, Taq activation at $95^{\circ} \mathrm{C}$ for $2 \mathrm{~min}$, followed by 45 cycles of denaturation at $95^{\circ} \mathrm{C}$ for $15 \mathrm{~s}$, and annealing at $55^{\circ} \mathrm{C}$ for $30 \mathrm{~s}$ ) [14]. Screening of respiratory specimens for noninfluenza viruses, including RSV, $h M P V$, parainfluenza viruses $1 / 2 / 3$, rhinoviruses (RV), adenoviruses (AdV), and bocaviruses (BoV) was performed using single-plex real-time PCR assays and an AgPath-ID One-Step RT-PCR kit (Applied Biosystems, Thermo Fisher Scientific, Waltham, MA, USA). Primers, probes, and thermocycling conditions used in the study were identical to those previously described $[15,16]$. Separate real-time RT-PCR assays for the RNAase-P gene were performed to verify RNA integrity and absence of PCR inhibition. For influenza A and B viruses, positive controls were provided by the International Reagent Resource, USA; for noninfluenza viruses, AmpliRun DNA/RNA amplification controls (Vircell, Granada, Spain) were used. Primer and probe sequences, as well as cycling parameters are shown in the online suppl. Table 1; see www.karger.com/doi/10.1159/000516821 for all online suppl. material.

\section{F Gene Sequencing}

A fragment of the hMPV F gene from hMPV-positive samples was amplified using the Qiagen One-Step RT-PCR kit (Qiagen, Hilden, Germany) and an Eppendorf Mastercycler instrument (Eppendorf, Stevenage, UK) with primers/protocol previously described (online suppl. Table) [17]. Amplified products corresponding to nucleotide positions 3,052-3,862 in the genome of the hMPV prototype strain NL00/1 (accession number: AF371337) were visualized via electrophoresis on a $2 \%$ ethidium bromidestained agarose gel. Amplicons were extracted and purified with a PureLink Quick Gel Extraction kit (Invitrogen, Thermo Fisher Scientific, Waltham, MA, USA). Purified PCR products were commercially sequenced (Microgen, Amsterdam) in both directions with the primer pair previously used for PCR. 
Table 1. Age distribution of hMPV infection among outpatients and inpatients

\begin{tabular}{llll}
\hline Age-group, years & Outpatients, $\%(n / N)$ & Inpatients, $\%(n / N)$ & Total, \% $(n / N)$ \\
\hline $0-4$ & $4.4(7 / 160)$ & $5.2(61 / 1,172)$ & $5.1(68 / 1,332)$ \\
$5-17$ & $4.2(1 / 24)$ & $2.7(4 / 146)$ & $2.9(5 / 170)$ \\
$18-64$ & $6.7(1 / 15)$ & $1.7(1 / 60)$ & $2.7(2 / 75)$ \\
$\geq 65$ & $0(0 / 25)$ & $3.3(8 / 240)$ & $3.0(8 / 265)$ \\
\hline Total & $4.0(9 / 225)$ & $4.6(74 / 1,617)$ & $4.5(83 / 1,842)$ \\
\hline
\end{tabular}

hMPV, human metapneumovirus.

\begin{abstract}
Phylogenetic Analysis
F gene nucleotide sequences of hMPV reference strains with known genotype identities were retrieved from GenBank using the basic local alignment search tool (http://blast.ncbi.nlm.nih.gov/ Blast.cgi). Bulgarian and reference sequences were aligned using the MUSCLE program embedded in molecular evolutionary genetics analysis software (MEGA, version 6.06; http://www.megasoftware.net/). The most suitable nucleotide substitution model, Tamura 3-parameter $(T-92+\mathrm{G})$ was determined using MEGA 6.06. A phylogenetic tree was constructed using the maximum likelihood method with 1,000 bootstrap replicates within MEGA 6.06 software. The study strains were genotyped based on clustering with sequences representing known genotypes.
\end{abstract}

Deduced Amino Acid Sequence Analysis

Deduced partial amino acid sequences of Bulgarian hMPV strains were generated by translating nucleotide sequences with the standard genetic code using MEGA software. To identify amino acid substitutions, the F protein sequences of the hMPV strains obtained in this study were aligned with the prototype NL00/1 strain.

Putative N-glycosylation sites were identified using the NetNGlyc 1.0 web server (http://www.cbs.dtu.dk/services/NetNGlyc) to predict the sequence motif $\mathrm{N}-\mathrm{X}-\mathrm{S} / \mathrm{T}$ (sequon), where $\mathrm{X}$ can be any amino acid except proline. Only sites with scores $>0.5$ were accepted as glycosylated.

\section{Statistics}

Patient age and gender, clinical syndromes, and incidence of each virus were compared using the $\chi^{2}$ or Fisher's exact tests for categorical variables. A $p$ value of $<0.05$ was considered statistically significant. Partial F gene nucleotide sequences of hMPV strains analyzed in this study were deposited in GenBank under the accession numbers MK577689-MK577699.

\section{Results}

\section{Patient Characteristics}

This prospective study was conducted from October 2016 to September 2019 and included 3 influenza seasons (2016/2017, 2017/2018, and 2018/2019), delineated as week 40 of the previous year to week 20 of the following year. A total of 1,842 patients presenting with ARI participated in the study: 498 in the first season, 500 in the second, and 743 in the third with 101 between seasons. In total, $225(12.2 \%)$ of these patients attended outpatient health-care centers, and 1,617 (87.8\%) were hospitalized. Patient ages ranged from 10 days to 91 years (median age 4.2 years); 1,007 (54.7\%) of the patients were males. There were 1,332 patients of $0-4$ years old, 170 were $5-17$ years old, 75 were $18-64$ years old, and 265 were $\geq 65$ years old (72.3, 9.2, 4.1, and $14.4 \%$, respectively).

\section{Viral Detection}

Viral respiratory pathogens were identified in 1,229 $(66.7 \%)$ of the 1,842 patients tested. Among the infected individuals, $1,042(84.8 \%)$ had monoinfection, $172(14 \%)$ were coinfected with 2 viruses, and 15 (1.2\%) were coinfected with 3 viruses. At least one respiratory virus was detected in $57.8 \%(130 / 225)$ of outpatients and $68 \%$ $(1,099 / 1,617)$ of inpatients $(p<0.05)$. hMPV was identified in $4.5 \%(83 / 1,842)$ of patient samples. The incidence rate of hMPV was lower than that of RSV, influenza $\mathrm{A}(\mathrm{H} 1 \mathrm{~N} 1)$ pdm09, influenza $\mathrm{A}(\mathrm{H} 3 \mathrm{~N} 2), \mathrm{RV}, \mathrm{AdV}$, and $\mathrm{BoV}(20.4,11.1$, $10.5,9.9,6.8$, and $5.3 \%$, respectively) but higher than that of parainfluenza viruses $1 / 2 / 3$ and influenza type B (4 and $3.3 \%$, respectively). The detection frequency of hMPV in the first, second, and third winter seasons was 5.4, 5.4, and $3.1 \%$, respectively, and was $4 \%$ in the periods between seasons. The incidence rates of hMPV infection among outpatients and inpatients were $4 \%(9 / 225)$ and $4.6 \%(74 / 1,617)$, respectively (Table 1), whereas the incidence rates of RSV infection were 12.3 and $32.8 \%(p<0.05)$, respectively. hMPV infections accounted for 6.9\% (9/130) and 6.7\% $(74 / 1,099)$ of total viral infections in outpatients and inpatients, respectively. hMPV monoinfection was found in 72 (86.7\%) patients, and 11 (13.3\%) of hMPV-positive patients were coinfected with another respiratory virus: AdV ( $n=$ $4)$, RV $(n=3)$, RSV $(n=2)$, influenza A(H3N2) $(n=1)$, and $\operatorname{BoV}(n=1)$. Among the studied respiratory viruses, the most common participants in mixed infections were AdV, $\mathrm{BoV}$, and RV, with proportions of coinfections at 58.1, 51, and $41.2 \%$, respectively. 


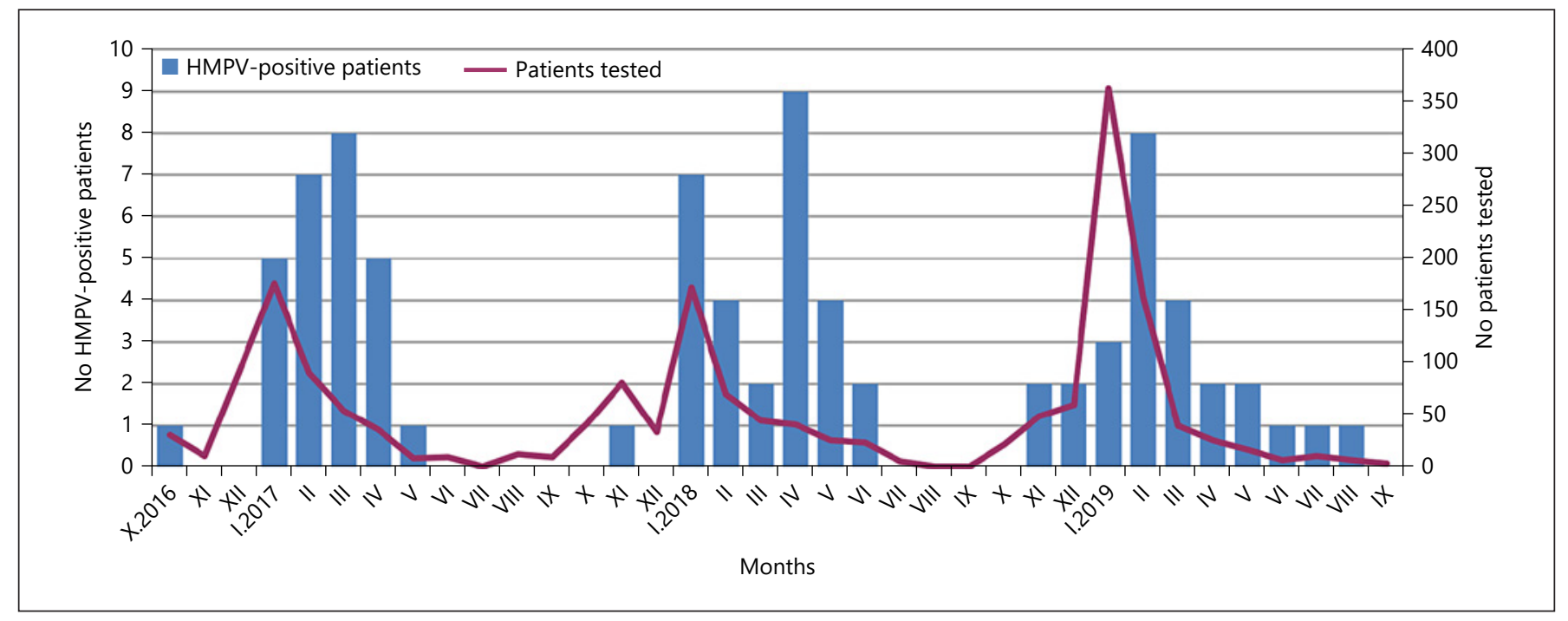

Fig. 1. Monthly distribution of hMPV-positive patients between October 2016 and September 2019. hMPV, human metapneumovirus.

Table 2. Clinical diagnosis in hMPV-infected patients according to age

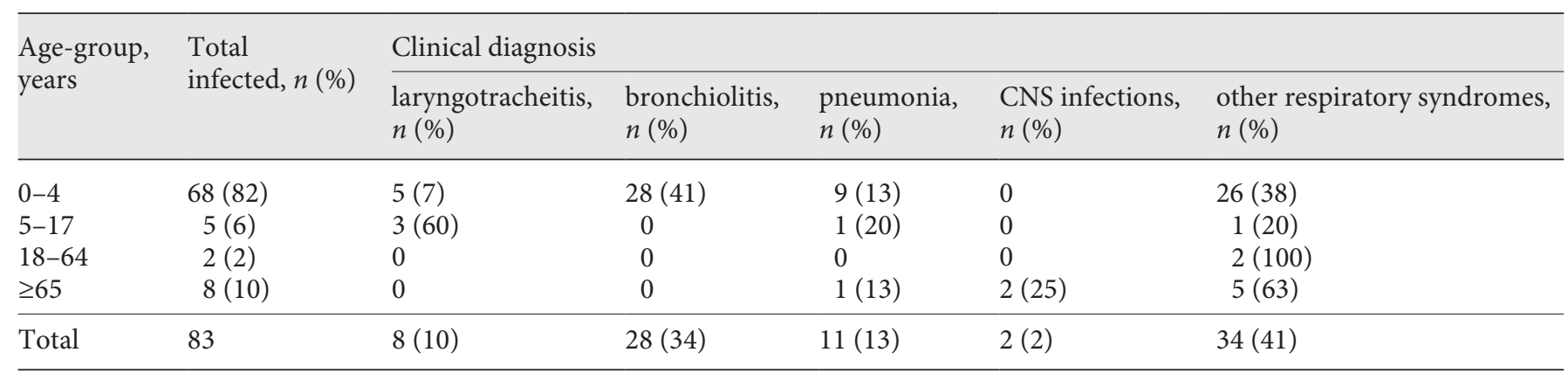

hMPV, human metapneumovirus; CNS, central nervous system.

hMPV infections were mainly detected between January and May (89.2\% of all identified infections) with few or no positive specimens in the autumn or summer months. During the study period, a higher incidence of hMPV was observed in April 2018, March 2017, and February 2019 (Fig. 1). In April 2018, the hMPV-positive rate reached 22\% of the collected samples. The periods of hMPV activity overlapped with those of RSV and influenza viruses.

\section{Age and Gender Distribution}

Viral respiratory infections were detected in individuals of all ages, and the positive rate in the 4 age-groups $0-4,5-17,18-64$, and $\geq 65$ years was $73.9 \%(983 / 1,332)$, $57.6 \%$ (98/170), 29.3\% (22/75), and 47.5\% (126/265), respectively. The age of patients infected with hMPV ranged between 1 month and 82 years. The incidence of hMPV

Human Metapneumovirus in Bulgaria, 2016-2019 was highest among the youngest age-group ( $0-4$ years) $(68 / 1,332,5.1 \%)$ and lowest among the young- and middle-aged adults (18-64 years) $(2 / 75,2.7 \%)$ (Table 1$)$. Children aged $0-4$ years represented $72.3 \%$ of the patients studied, but accounted for $81.9 \%(68 / 83)$ of the hMPVpositive cases $(p=0.0586)$. In this age-group, the median age of hMPV-positive patients (26.2 months) was higher than that of RSV-positive patients (15 months). hMPV coinfections were only detected in children aged 0-4 years. Among the hMPV-positive subjects, 43 (51.8\%) were males, and $40(48.2 \%)$ were females $(p=0.7)$.

\section{Clinical Characteristics}

The contribution of hMPV and the other respiratory viruses tested in the development of the most commonly diagnosed clinical complications - laryngotracheitis, 


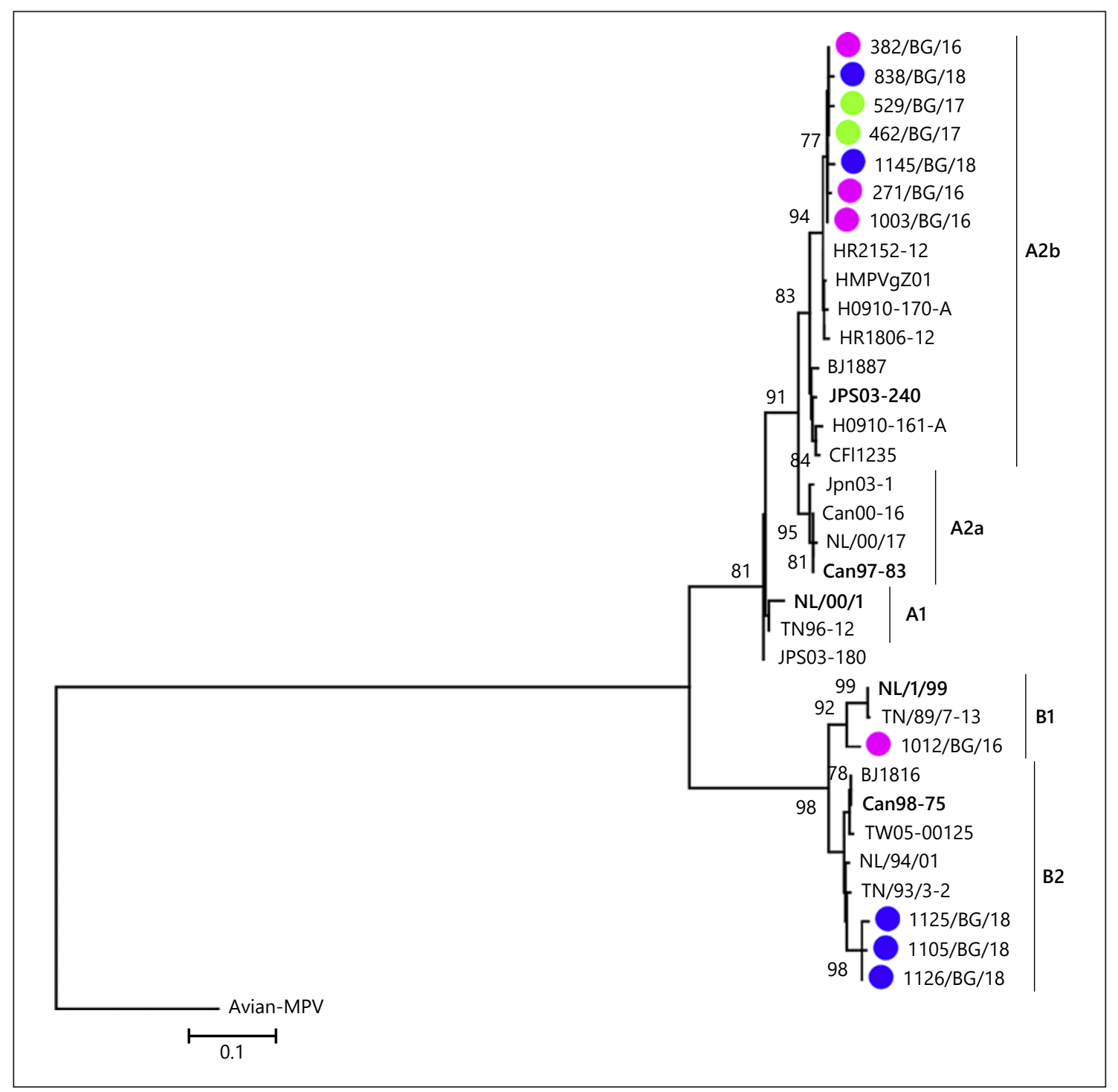

Fig. 2. Phylogenetic analysis based on the $F$ gene sequences of hMPV strains. A phylogenetic tree was constructed using the maximum likelihood method and the Tamura 3-parameter (T-92+ G) model with 1,000 bootstrap replicates within MEGA 6.06 software. Avian MPV C was included and used as outgroup. Only bootstrap values $\geq 70 \%$ are displayed at the branch nodes. The scale bar indicates the number of nucleotide substitutions per site. The Gen-

bronchiolitis, pneumonia, and the central nervous system involvement (febrile seizures, cerebral edema, aseptic meningitis, and encephalopathy) was analyzed. The proportion of detected respiratory viruses among children aged $0-4$ years diagnosed with these conditions was $79.6 \%$ (90/113), 82.9\% (287/346), 67.2\% (125/186), and 58.1\% (25/43), respectively. hMPV ranked fourth among the causative agents of bronchiolitis $(8.1 \%, 28 / 346)$ following
Bank names of the strains are shown in the phylogenetic trees. The genotypes are denoted by lines on the right side. Reference sequences representing genotypes $\mathrm{A} 1, \mathrm{~A} 2 \mathrm{a}, \mathrm{A} 2 \mathrm{~b}, \mathrm{~B} 1$, and $\mathrm{B} 2$ are in bold text. Bulgarian hMPV strains detected during the 2016/2017, $2017 / 2018$, and 2018/2019 seasons are indicated in purple, green, and blue circles, respectively. hMPV, human metapneumovirus; MEGA, molecular evolutionary genetics analysis software.

RSV, RV, and BoV $(42.2,16.5$, and $11 \%$, respectively) and sixth among the causes of pneumonia $(4.8 \%, 9 / 186)$, following RSV, AdV, RV, influenza A(H3N2), and A(H1N1) pdm09 (28.5, 9.7, 9.7, 9.1, and 5.9\%, respectively). Seven (7/11, 63.6\%) children who had coinfections of hMPV with other respiratory virus were diagnosed with bronchiolitis or pneumonia. hMPV was detected in $2.2 \%$ $(2 / 91)$ and $3.2 \%(2 / 63)$ of cases of pneumonia and central 


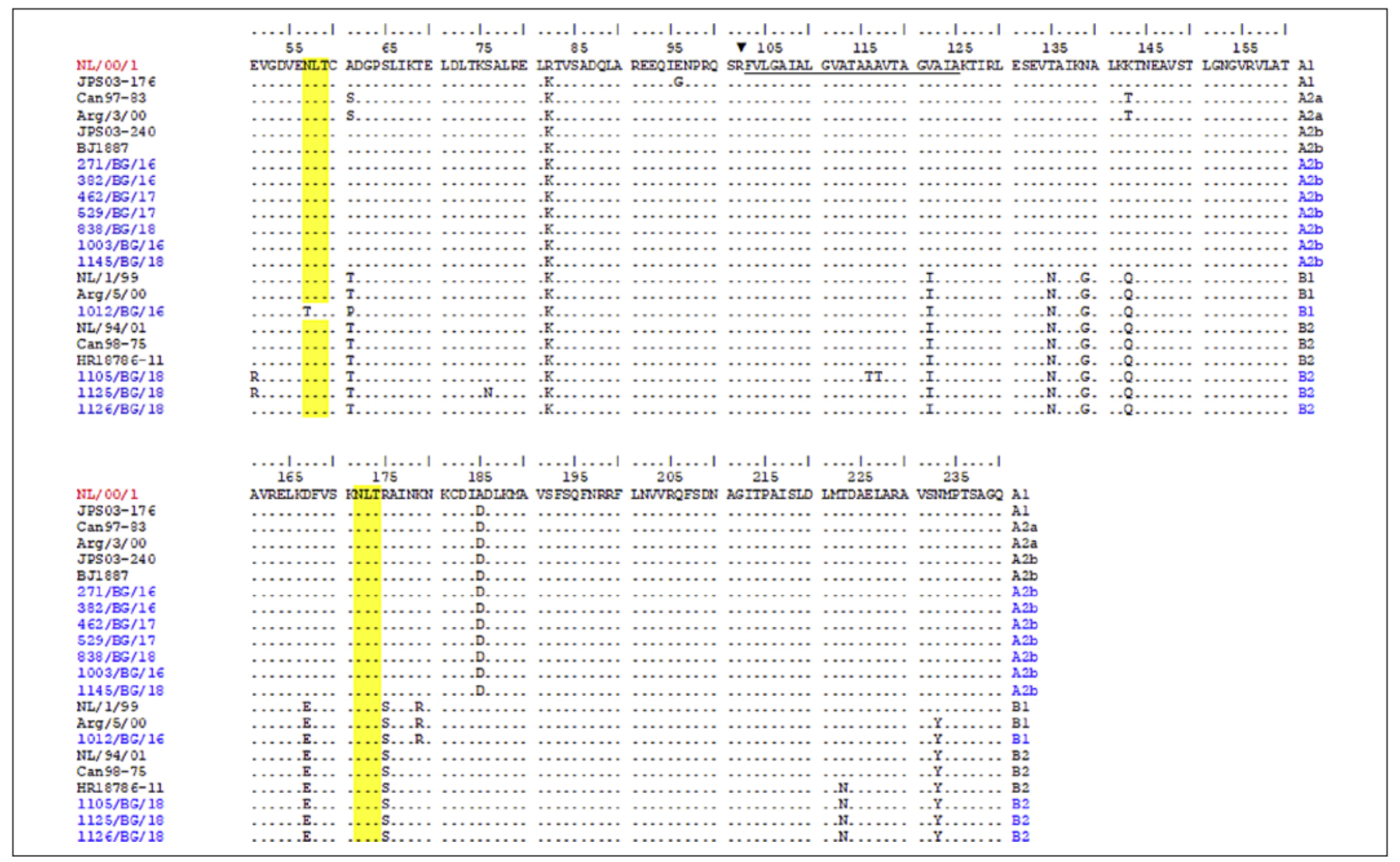

Fig. 3. Deduced amino acid alignment of partial F protein of hMPV strains. The alignment is shown relative to the sequence of reference NL/00/1 strain (GenBank accession number: AF371337). The Bulgarian hMPV strains are indicated in blue. Identical residues

nervous system infections, respectively, in patients aged $\geq 5$ years (Table 2). No hMPV-positive patients enrolled in this study were treated in intensive care units.

\section{Phylogenetic Analysis of $h M P V$}

Partial F gene sequences, deposited to GenBank, were obtained from $11 \mathrm{hMPV}$-positive clinical samples. The remaining positive samples had a poor or failed PCR amplification or sequencing probably because of a low viral load and the greater sensitivity of real-time PCR compared with conventional PCR. The mean amplification cycle threshold $\left(c_{t}\right)$ obtained for the successfully sequenced hMPV-positive samples was 23.49 versus 30.79 for the unsequenced. The sequences were obtained from hospitalized children aged 0-4 years from different regions of the country who had ALRI. The phylogenetic analysis showed circulation of the 2 major groups (A and B), represented by genotypes A2b, B1, and B2 (Fig. 2). Seven sequenced Bulgarian hMPV strains belonged to the are identified as dots. The fusion peptide is underlined. The protease cleavage site is indicated with a black triangle. Yellow shading highlights the predicted N-glycosylation sites. hMPV, human metapneumovirus.
A2b genotype, one to the $\mathrm{B} 1$ genotype, and 3 to the $\mathrm{B} 2$ genotype. Neither the A1 nor A2a genotypes were detected in our study population. Viruses of the A2b genotype were identified during each study season; a genotype B1 virus was identified in the $2016 / 2017$ season, and genotype B2 viruses were identified in the 2018/2019 season.

The hMPV A2b strains identified in this study were 94.5-98.9\% homologous at the nucleotide level and 96.9$99.4 \%$ homologous at the amino acid level. The B2 strains had $93.2-96.3 \%$ and $95.4-97.8 \%$ nucleotide and amino acid sequence homology, respectively.

\section{Deduced Amino Acid Sequence Analysis}

We aligned the deduced amino acid sequences of 11 Bulgarian hMPVs and compared these with those of the prototype strain NL00/1 identified by van den Hoogen et al. [18] and also with hMPV strains detected in other countries (Fig. 3). The Bulgarian F sequences included 190 amino acids, representing $35.3 \%$ of the entire $\mathrm{F}$ pro- 
tein sequence (539 residues) [7]. In A2b genotype sequences, amino acid substitutions were detected at 2 positions: R82K (in subunit F2) and A185D (in subunit F1), which were also present in the A2b sequences detected in other countries. Further amino acid substitutions were detected in the genotype B strains. A total of 9 amino acid substitutions were identified in the Bulgarian genotype B2 sequences, as well as in the other analyzed sequences of the same genotype: A61T and R82K (in subunit F2); V122I (in the fusion peptide); T135N, N139G, K143Q, and D167E (in heptad repeat A [HRA]); and R175S, T223N, and N233Y (in subunit F1). The Bulgarian B1 genotype sequence carried additional substitutions compared with the B2 sequences: N57T (in subunit F2) and K179R (in subunit F1) [19]. The N57T substitution resulted in the loss of the N-glycosylation site (NLT). This sequence lacked the T223N substitution found in the genotype B2 strains. In the Bulgarian hMPV sequences, no substitutions were identified in antigenic regions (epitopes) 2 (residues 132 and 152), 3 (residues 177-179), 4 (residues 238-245), and 5-6 (residues 386-397) with the exception of the K179R substitution in the antigenic region 3 of the genotype B1 sequence [20]. All but one analyzed Bulgarian sequence possessed 2 potential $\mathrm{N}$-linked glycosylation sites, at positions 57 (NLT) and 172 (NLT).

\section{Discussion}

Here, we explored the prevalence and genetic characteristics of hMPV in an all age population in Bulgaria over 3 consecutive seasons, together with the epidemiological and clinical features of hMPV infections. Among the 12 respiratory viruses studied, hMPV had a moderate incidence rate and ranked seventh highest after that of RSV, influenza A viruses, $\mathrm{RV}, \mathrm{AdV}$, and $\mathrm{BoV}$. The overall detection rate of hMPV was $4.5 \%$, with a significant difference between seasons $(5.4,5.4$, and $3.1 \%)$. The hMPVpositive rate in this study was lower than that reported in Italy (9.3\%), Spain (5.5\%), Croatia (5.66\%), Romania (12.5\%), Belgium (7.3\%), Norway (9.4\%), the USA (6\%), and India $(5.11 \%)$ [21-28], but was higher than that in other studies in the USA (3.6\%), Germany (3\%), China (1.7\%), Cambodia (1.7\%), and Malaysia (2.2\%) [29-33]. Earlier studies in Bulgaria reported a hMPV prevalence between 6.9 and $16 \%$ among children younger than 5 years $[10,11]$. The prevalence of hMPV infections varies in individual studies and countries because of differences in the population studied, methodology used, climate, and time period of the study [34]. Here, the incidence rate of hMPV among outpatients and inpatients was similar (4 and 4.6\%, respectively), whereas German researchers recorded a significantly higher incidence of hMPV infections in hospitalized patients in comparison with outpatients (11.9 vs. 6.9\%) [30]. The proportion of hMPV coinfections in study population (13.3\%) was relatively low compared with that of $\mathrm{AdV}, \mathrm{BoV}$, and RV coinfections (58.1, 51, and $41.2 \%$, respectively). A similar frequency of hMPV coinfections (14.6\%) has been reported in other countries [35]. However, several authors also reported a significantly higher proportion of hMPV coinfections 58 [36] and 63.9\% [9]. hMPV infection in Bulgaria had a clear seasonality with the highest frequency of occurrence in winter and spring months, which was consistent with data from other countries with temperate climates [22, $27,37]$. In our surveillance period, hMPV was circulating mainly from January to May and co-circulated with RSV and influenza viruses. In agreement with many studies, the incident rate of hMPV was highest in the youngest age-groups ( $0-4$ years), and $81.9 \%$ of all hMPV cases occurred in this age-group [27, 38]. Among children aged 0-4 years with ARI, the median age of the hMPV-positive subjects was higher (26.2 months) than that of the RSVpositive subjects ( 15 months), which agrees with previous reports [26]. Due to the higher incidence of RSV infection, children are probably first exposed to this virus at an earlier age. We did not find any influence of the gender of the patients on hMPV infection, in contrast to other reports [33]. Several studies have documented that hMPV is the second most common pathogen of the lower respiratory tract in infants and young children after RSV, and is the major cause of bronchiolitis and pneumonia $[3,22$, 39-42]. In this study, the detection rate of hMPV ranked fourth and sixth among the causative agents of bronchiolitis and pneumonia, respectively, in children aged 0-4 years.

Genetic variability of the hMPV F gene was analyzed since the F protein is highly immunogenic, induces protective immunity, and is therefore a potential target for vaccines, monoclonal antibodies, and antiviral drugs [7]. Amino acid changes in the antigenic sites of the hMPV protein $\mathrm{F}$ could help avoid the immunity generated by previous infection. Despite the small number of sequenced strains, we identified the presence of 3 (A2b, B1, and B2) of the 5 globally circulating genotypes during the study period. However, we could not determine with certainty how many genotypes circulated in a particular season and which of them predominated. Simultaneous circulation of several hMPV genotypes within one epidemic season and replacement of the predominant genotype every $1-3$ years 
in a given population have been reported worldwide [33, 35]. This replacement of predominant genotypes probably results from adaptive immunity of population against the genotype that was prevalent in the country in the preceding year. Similar to other studies, our results indicated that the $\mathrm{F}$ gene/protein is relatively conserved but has enough variability to permit distinction between genotypes $[43,44]$. Amino acid substitutions were identified at several positions of the $\mathrm{F}$ protein including at one antigenic site and in 2 functional domains, the fusion peptide and HRA. The HRA and HRB domains participate in protein folding during the fusion process [6]. hMPVs from genotype $B$ carried a larger number of amino acid substitutions (9-11) compared with those in the A2b genotype (2) in agreement with other studies [32]. The N-linked glycosylation sites at positions 57 and 172, identified in our study, were located in an antigenic site near the glycoprotein trimer apex, and the glycans in these positions could prevent antibodies recognition [45]. The gradual accumulation of amino acid changes and the glycosylation of the hMPV F protein with N-linked sugars are important mechanisms used by viruses to escape preexisting immunity [28]. In this regard, molecular characterization of the $\mathrm{G}$ gene/protein would provide more comprehensive information on the genetic variability and evolutionary dynamics of this pathogen.

In summary, the present study demonstrated a high incidence of viral respiratory infections and moderate incidence of hMPV infection among Bulgarian patients with ARIs. Our results indicated that hMPV is a significant causative agent of bronchiolitis and pneumonia in children aged $0-4$ years. The study highlighted the necessity of routine laboratory-based surveillance and continuous monitoring of the genetic characteristics of this pathogen for the early detection of novel genetic variants that have increased virulence or epidemic potential. Our study has a significant limitation. The small number of sequenced samples limited our ability to more fully characterize the genotypes circulating in Bulgaria during the study period. A larger number of sequenced samples is needed in future studies to get a complete picture of the molecular epidemiology of the hMPV in Bulgaria. Information regarding genetic diversity of hMPV in different countries will be important for the development of a globally effective vaccine.

\section{Acknowledgments}

The authors are grateful to all of the nurses and clinicians involved in this study for their help in collecting clinical specimens and patient information.

\section{Statement of Ethics}

This research was conducted ethically in accordance with the World Medical Association Declaration of Helsinki for experiments involving humans http://www.healthscience.net/resources/ declaration-of-helsinki. The study protocol was approved by the Institutional Review Board/Institutional Ethics Committee of the National Centre of Infectious and Parasitic Diseases (number: IRB 00006384). Written informed consent to participate in the study was obtained from participants (or their parents/legal guardians) before specimen collection and testing.

\section{Conflict of Interest Statement}

The authors have no conflicts of interest to declare.

\section{Funding Sources}

This work was supported by financial grant from the National Science Foundation of Bulgaria (Project DH 13-15/December 20, 2017).

\section{Author Contributions}

Conceived and designed the experiments: N.K.; performed the experiments: N.K., S.A., I.Tr., S.V., I.G., I.Tz., S.M., I.A., and P.P.; analyzed the data: N.K., I.Tr., and I.Tz.; wrote the paper: N.K. All authors read and approved the final manuscript.

\section{References}

1 van den Hoogen BG, van Doornum GJ, Fockens JC, Cornelissen JJ, Beyer WE, de Groot $\mathrm{R}$, et al. Prevalence and clinical symptoms of human metapneumovirus infection in hospitalized patients. J Infect Dis. 2003;188:15717

2 Boivin G, Abed Y, Pelletier G, Ruel L, Moisan $D$, Côté $S$, et al. Virological features and clinical manifestations associated with human metapneumovirus: a new paramyxovirus responsible for acute respiratory-tract infec- tions in all age groups. J Infect Dis. 2002; 186(9):1330-4.

3 Williams JV, Harris PA, Tollefson SJ, Halburnt-Rush LL, Pingsterhaus JM, Edwards $\mathrm{KM}$, et al. Human metapneumovirus and lower respiratory tract disease in otherwise healthy infants and children. $N$ Engl J Med. 2004;350(5):443-50.

4 Wang X, Li Y, Deloria-Knoll M, Madhi SA, Cohen C, Ali A, et al. Global burden of acute lower respiratory infection associated with human metapneumovirus in children under 5 years in 2018: a systematic review and modelling study. Lancet Glob Health. 2021 Jan;9(1):e33-43.

5 Lefkowitz EJ, Dempsey DM, Hendrickson RC, Orton RJ, Siddell SG, Smith DB. Virus taxonomy: the database of the international committee on taxonomy of viruses (ICTV). Nucleic Acids Res. 2018;46:D708-17.

6 Feuillet F, Lina B, Rosa-Calatrava M, Boivin G. Ten years of human metapneumovirus research. J Clin Virol. 2012;53(2):97-105. 
7 Melero JA, Mas V. The pneumovirinae fusion (F) protein: a common target for vaccines and antivirals. Virus Res. 2015;209:128-35.

8 Regev L, Meningher T, Hindiyeh M, Mendelson E, Mandelboim M. Increase human metapneumovirus mediated morbidity following pandemic influenza infection. PLoS One. 2012;7:e34750.

9 Xie Z, Xu J, Ren Y, Cui A, Wang H, Song J, et al. Emerging human metapneumovirus gene duplication variants in patients with severe acute respiratory infection, China, 20172019. Emerg Infect Dis. 2021;27(1):275-7

10 Pavlova S, Hadzhiolova T, Abadjieva P, Kotseva R. Application of RT-PCR for diagnosis of respiratory syncytial virus and human metapneumovirus infections in Bulgaria, 2006-7 and 2007-8. Euro Surveill. 2009; 14(23): 19233 .

11 Korsun N, Angelova S, Tzotcheva I, Georgieva I, Lazova S, Parina S, et al. Prevalence and genetic characterisation of respiratory syncytial viruses circulating in Bulgaria during the 2014/15 and 2015/16 winter seasons. Pathog Glob Health. 2017;111(7):351-61.

12 Korsun N, Angelova S, Trifonova I, Georgieva I, Voleva S, Tzotcheva I, et al. Viral pathogens associated with acute lower respiratory tract infections in children younger than 5 years of age in Bulgaria. Braz J Microbiol. 2019;50(1):117-25.

13 Korsun N, Angelova S, Trifonova I, Voleva V, Grigorova I, Tzotcheva I, et al. Predominance of ON1 and BA9 genotypes of respiratory syncytial virus in Bulgaria, 2016-2018. J Med Virol. 2021;93(6):3401-11.

14 Shu B, Wu KH, Emery S, Villanueva J, Johnson R, Guthrie E, et al. Design and performance of the CDC real-time reverse transcriptase PCR swine flu panel for detection of $2009 \mathrm{~A}(\mathrm{H} 1 \mathrm{~N} 1)$ pandemic influenza virus. J Clin Microbiol. 2011;49:2614-9.

15 Kodani M, Yang G, Conklin LM, Travis TC, Whitney CG, Anderson LJ, et al. Application of TaqMan low-density arrays for simultaneous detection of multiple respiratory pathogens. J Clin Microbiol. 2011;49(6):2175-82.

16 Lu X, Chittaganpitch M, Olsen SJ, Mackay IM, Sloots TP, Fry AM, et al. Real-time PCR assays for detection of bocavirus in human specimens. J Clin Microbiol. 2006;44(9):3231-5.

17 Ludewick HP, Abed Y, van Niekerk N, Boivin G, Klugman KP, Madhi SA. Human metapneumovirus genetic variability, South Africa. Emerg Infect Dis. 2005;11(7):1074-8.

18 van den Hoogen BG, de Jong JC, Groen J, Kuiken T, de Groot R, Fouchier RA, et al. A newly discovered human pneumovirus isolated from young children with respiratory tract disease. Nat Med. 2001;7(6):719-24.

19 Yang CF, Wang CK, Tollefson SJ, Piyaratna $\mathrm{R}$, Lintao LD, Chu M, et al. Genetic diversity and evolution of human metapneumovirus fusion protein over twenty years. Virol J. 2009;6:138.

20 Ulbrandt ND, Ji H, Patel NK, Barnes AS, Wilson S, Kiener PA, et al. Identification of antibody neutralization epitopes on the fusion protein of human metapneumovirus. J Gen Virol. 2009;89(Pt 12):3113-8.

21 Apostoli P, Zicari S, Lo Presti A, Ciccozzi M, Ciotti M, Caruso A, et al. Human metapneumovirus-associated hospital admissions over five consecutive epidemic seasons: evidence for alternating circulation of different genotypes. J Med Virol. 2012;84(3):511-6.

22 García-García ML, Calvo C, Rey C, Díaz B, Molinero MD, Pozo F, et al. Human metapnuemovirus infections in hospitalized children and comparison with other respiratory viruses. 2005-2014 prospective study. PLoS One. 2017;12(3):e0173504.

23 Jagušić M, Slović A, Ljubin-Sternak S, MlinarićGalinović G, Forčić D. Genetic diversity of human metapneumovirus in hospitalized children with acute respiratory infections in Croatia. J Med Virol. 2017;89(11):1885-93.

24 Tecu C, Mihai ME, Alexandrescu VI, Orăşeanu D, Zapucioiu C, Ivanciuc AE, et al. Single and multipathogen viral infections in hospitalized children with acute respiratory infections. Roum Arch Microbiol Immunol. 2013;72(4):242-9.

25 Cattoir L, Vankeerberghen A, Boel A, Van Vaerenbergh K, De Beenhouwer H. Epidemiology of RSV and hMPV in Belgium: a 10-year follow-up. Acta Clin Belg. 2019;74(4):229-35.

26 Moe N, Krokstad S, Stenseng IH, Christensen A, Skanke LH, Risnes KR, et al. Comparing human metapneumovirus and respiratory syncytial virus: viral co-detections, genotypes and risk factors for severe disease. PLoS One. 2017;12(1):e0170200

27 Edwards KM, Zhu Y, Griffin MR, Weinberg GA, Hall CB, Szilagyi PG, et al. Burden of human metapneumovirus infection in young children. N Engl J Med. 2013;368(7):633-43.

28 Agrawal AS, Roy T, Ghosh S, Chawla-Sarkar $M$. Genetic variability of attachment $(\mathrm{G})$ and fusion (F) protein genes of human metapneumovirus strains circulating during 2006-2009 in Kolkata, Eastern India. Virol J. 2011;8:67.

29 Haynes AK, Fowlkes AL, Schneider E, Mutuc JD, Armstrong GL, Gerber SI. Human metapneumovirus circulation in the United States, 2008 to 2014. Pediatrics. 2016;137(5): e20152927.

30 Reiche J, Jacobsen S, Neubauer K, Hafemann S, Nitsche A, Milde J, et al. Human metapneumovirus: insights from a ten-year molecular and epidemiological analysis in Germany. PLoS One. 2014;9(2):e88342.

31 Li J, Wang Z, Gonzalez R, Xiao Y, Zhou H, Zhang J, et al. Prevalence of human metapneumovirus in adults with acute respiratory tract infection in Beijing, China. J Infect. 2012;64(1):96-103.

32 Arnott A, Vong S, Sek M, Naughtin M, Beauté J, Rith S, et al. Genetic variability of human metapneumovirus amongst an all ages population in Cambodia between 2007 and 2009. Infect Genet Evol. 2013;15:43-52.

33 Chow WZ, Chan YF, Oong XY, Ng LJ, Nor'E SS, Ng KT, et al. Genetic diversity, seasonality and transmission network of human metapneumovirus: identification of a unique sub- lineage of the fusion and attachment genes. Sci Rep. 2016;6:27730.

34 Lefebvre A, Manoha C, Bour JB, Abbas R, Fournel I, Tiv M, et al. Human metapneumovirus in patients hospitalized with acute respiratory infections: a meta-analysis. J Clin Virol. 2016;81:68-77.

35 Kong W, Wang Y, Zhu H, Lin X, Yu B, Hu Q, et al. Circulation of human metapneumovirus among children with influenza-like illness in Wuhan, China. J Med Virol. 2016;88(5):774-81.

36 Xiao NG, Zhang B, Xie ZP, Zhou QH, Zhang RF, Zhong LL, et al. Prevalence of human metapneumovirus in children with acute lower respiratory infection in Changsha, China. J Med Virol. 2013;85(3):546-53.

37 Fillatre A, François C, Segard C, Duverlie G, Hecquet D, Pannier C, et al. Epidemiology and seasonality of acute respiratory infections in hospitalized children over four consecutive years (2012-2016). J Clin Virol. 2018;102:27-31.

38 Tsagarakis NJ, Sideri A, Makridis P, Triantafyllou A, Stamoulakatou A, Papadogeorgaki E. Age-related prevalence of common upper respiratory pathogens, based on the application of the filmArray respiratory panel in a tertiary hospital in Greece. Medicine. 2018;97(22):e10903.

39 Anderson EJ, Simões EA, Buttery JP, Dennehy $\mathrm{PH}$, Domachowske JB, Jensen K, et al. Prevalence and characteristics of human metapneumovirus infection among hospitalized children at high risk for severe lower respiratory tract infection. J Pediatric Infect Dis Soc. 2012;1(3):212-22.

40 Xiao NG, Zhang B, Xie ZP, Zhou QH, Zhang RF, Zhong LL, et al. Prevalence of human metapneumovirus in children with acute lower respiratory infection in Changsha, China. J Med Virol. 2013;85(3):546-53.

41 Hahn A, Wang W, Jaggi P, Dvorchik I, Ramilo O, Koranyi K, et al. Human metapneumovirus infections are associated with severe morbidity in hospitalized children of all ages. Epidemiol Infect. 2013;141(10):2213-23.

42 Wong-Chew RM, García-León ML, Noyola DE, Perez Gonzalez LF, Gaitan Meza J, Vilaseñor-Sierra $A$, et al. Respiratory viruses detected in Mexican children younger than 5 years old with community-acquired pneumonia: a national multicenter study. Int J Infect Dis. 2017;62:32-8.

43 Papenburg J, Carbonneau J, Isabel S, Bergeron MG, Williams JV, De Serres G, et al. Genetic diversity and molecular evolution of the major human metapneumovirus surface glycoproteins over a decade. J Clin Virol. 2013; 58(3):541-7.

44 van den Hoogen BG, Herfst S, Sprong L, Cane PA, Forleo-Neto E, de Swart RL, et al. Antigenic and genetic variability of human metapneumoviruses. Emerg Infect Dis. 2004; 10(4):658-66.

45 Battles MB, Más V, Olmedillas E, Cano O, Vázquez M, Rodríguez L, et al. Structure and immunogenicity of pre-fusion-stabilized human metapneumovirus F glycoprotein. Nat Commun. 2017;8(1):1528. 